Introduction: Exercise-induced asthma (EIA) is a common respiratory disorder. The mechanism of airway narrowing is not simple, but airway drying and cooling evidently play a major role. The aim of this study was to investigate how closely the airway narrowing observed in EIA depends on ambient temperature and humidity, and whether the dependence on humidity is greater than on temperature.

Material and Methods: The population consisted of 1,809 army conscripts admitted to the Central Military Hospital in Helsinki, in order to assess their fitness for military service or to diagnose asthma. The maximum decrease in peak expiratory flow (DPEF\%) was measured 30 minutes after an eightminute outdoor running test, with the heart rate at $85 \%$ of the predicted maximum. The dependence of DPEF\% on temperature and humidity was analysed by a linear regression model. Statistical significance was tested using the t-test.

Results: The dependence of DPEF\% on temperature and humidity was negative and significant statistically $(p<0.001)$. The regression coefficient with respect to absolute air humidity was -1.026 and with respect to air temperature was -0.327 . The difference was significant statistically.

- $\mathrm{DPEF} \%=23.907-1.026 \mathrm{x}$ absolute air humidity, $p<0.001$.

- DPEF\% $=20.347-0.327 \times$ air temperature, $p<0.001$.

The DPEF\% was dependent most clearly on temperature and humidity when the ambient temperature was below $-4^{\circ} \mathrm{C}$ and humidity below $4 \mathrm{gH}_{2} \mathrm{O} / \mathrm{kg}$.

Conclusion: Air temperature, and more obviously air humidity, have a significant influence on airway narrowing in EIA, and should be taken into account when EIA tests are performed on runners in the outdoors.

Keywords: asthma, exercise-induced; humidity; peak expiratory flow rate; temperature; weather

Prebosp Disast Med 2002;17:s5.

Cold-Allodynia and Alterations in Thin- and ThickFibre Function after Cold Injuries in Military Coastal Rangers

Jorum E, Opstad PK

Laboratory of Neurophysiology, Department of Neurology, National Hospital, Oslo; Norwegian Defense Research Establishment, Division for Material and Protection, Kjeller, Norway

Introduction: Cold allodynia and hyperalgesia are typical findings in patients with neuropathic pain of different origins. The neurophysiological mechanisms are unknown. Cold allodynia and hyperalgesia also are observed as sequelae in subjects who previously have been exposed to cold injuries.

Material and Methods: The subjects investigated for this study were 26 coastal rangers and officers who participated in a 5-day military training course, in small, open vessels along the coast of Helgeland in January 1998. All soldiers were exposed to the same type of cold: wet and cloudy the first 2 days, followed by cold and wind the remaining days. The first investigation of the soldiers was performed 2-3 months after the cold injury. All soldiers had a thorough clinical investigation, including a neurography, an investigation of thin fiber function by Thermotest (Somedic), a measurement of subcutaneous fat, and maximal oxygen uptake.

Results: Six subjects were without symptoms and had normal neurological and neurophysiological findings. The other 20 had different types of sensory disturbances. Compared to a normal control group from the Norwegian Military Academy, the rest of the subjects had neurophysiological findings of both thick and thin fiber neuropathy. There was no significant alteration in the heat pain threshold. The most significant and interesting finding was the reduction in the cold pain threshold in the feet (cold allodynia). A one and two year follow-up has demonstrated that the thin and thick fiber neuropathy healed completely, whereas cold allodynia still persisted in some of the subjects. Ischaemic blockade of the nerve fibers indicates that the cold allodynia is mediated through the Ad-fibers.

Conclusion: Soldiers with persistent symptoms after 2 years experienced pain when exposed to cold at higher temperature than normal. Cold allodynia, as demonstrated by the Thermotest, is the neurophysiological finding of relevance to this symptom. Therefore, assessment of thin-fiber function is of great value in evaluating patients with sequelae following cold injuries.

Definition: Allodynia is the pain response to stimuli that normally are not painful.

Keywords: allodynia; cold; hyperalgesia; injuries; neuropathy; sequelae; thermotest

Prehosp Disast Med 2002;17:s5.

\section{Stress-Related Anterior Lower Leg Pain: Evaluation with MRI and Intracompartmental Pressure Measurement}

\section{Kiuru MJ, ${ }^{1,2}$ Mäntysaari $M J,^{1}$ Piblajamäki $\mathrm{HK}^{3}$ \\ Abovuo $J A^{2}$}

1. Research Institute of Military Medicine, Helsinki, Finland

2. Department of Radiology, Helsinki University Centra 1 Hospital, Helsinki, Finland

3. Department of Surgery, Central Military Hospital, Helsinki, Finland

Purpose: To assess the feasibility of MRI and anterior tibial compartment pressure measurement in evaluating the causes of stress-related anterior lower leg pain.

Materials and Methods: This series was composed of 24 consecutive conscripts suffering from stress-related, anterior, lower leg pain. Twenty patients had bilateral symptoms. The patients underwent clinical examination, MR imaging, and intra-compartmental pressure measurement. The intracompartmental pressure was measured in the anterior tibial compartment using a standardized protocol. Intra-compartmental pressure values higher than $40 \mathrm{mmHg}$ were considered pathological.

Results: As compared with the intracompartmental pressure measurement, $50 \%$ of the patients had a false-positive clinical diagnosis of chronic exertional compartment syndrome (CECS). The duration of symptoms was longer in the patients with CECS than in those without $(p<0.01)$. MR imaging revealed abnormal findings in 35 symptomatic legs, the remaining 9 symptomatic legs were normal. In 33 legs, there was a stress fracture, and two legs had traction periostitis. There were no significant differences in the bone findings between the patients with CECS and those without $(p>0.05)$. Conclusions: Stress-related anterior lower leg pain may result from CECS, stress fracture, and traction periostitis. The clinical diagnosis is not reliable. CECS and stress fracture or traction periostitis may occur separately or simultaneously.

Keywords: compartmental pressures; leg; MRI; pain; periostitis; stress fracture

Prebosp Disast Med 2002;17:s6. 